\title{
Effect of Permeability and Piezomagnetic Coefficient on Magnetostrictive/Piezoelectric Laminate Composite
}

\author{
Zhiyi Wu, Yumei Wen*, Ping Li, Jin Yang, and Xianzhi Dai \\ College of Optoelectronic Engineering, Chongqing University, Chongqing City, 400044, P.R. China \\ The Key Laboratory for Optoelectronic Technology \& Systems, Ministry of Education, China
}

(Received 25 February 2011, Received in final form 10 April 2011, Accepted 12 May 2011)

\begin{abstract}
The magnetostrictive material is magnetized in magnetic field and produces a nonuniform demagnetizing field inside and outside it. The demagnetization is decided by the permeability of magnetostrictive material and its size. The magnetoelectric performances are determined by the synthesis of the applied and demagnetizing fields. An analytical model is proposed to predict the magnetoelectric voltage coefficient (MEVC) of magnetostrictive/piezoelectric laminate composite using equivalent circuit method, in which the nonuniform demagnetizing field is taken into account. The theoretical and experimental results indicate that the MEVC is positively connected with the permeability and the piezomagnetic coefficient of magnetostrictive material. To obtain the maximum MEVC, both the permeability and the piezomagnetic coefficient of magnetostrictive material should be taken into account in selecting the suitable magnetostrictive material.
\end{abstract}

Keywords : magnetoelectric performance, magnetostrictive material, permeability, piezomagnetic coefficient

\section{Introduction}

Composites consisting of magnetostrictive Terfenol-D, $\mathrm{Ni}_{1-\mathrm{x}} \mathrm{Co}_{\mathrm{x}} \mathrm{Fe}_{2} \mathrm{O}_{4}, \mathrm{Co}_{1-\mathrm{x}} \mathrm{Zn}_{\mathrm{x}} \mathrm{Fe}_{2} \mathrm{O}_{4}, \mathrm{FeNi}$, or Permendur layers laminated with piezoelectric $\mathrm{Pb}(\mathrm{Zr}, \mathrm{Ti}) \mathrm{O}_{3} \quad$ (PZT) or $\mathrm{Pb}\left(\mathrm{Mg}_{1 / 3} \mathrm{Nb}_{2 / 3} \mathrm{O}_{3}-\mathrm{PbTiO}_{3}\right)(\mathrm{PMNT})$ ones are known to have significant magnetoelectric (ME) effects, offering potential application in magnetic field sensors, energy harvesting and transformers [1]. In previous literature [2,3], to maximize the magnetoelectric voltage coefficient (MEVC), much attention is focused on selecting materials with strong magnetostrictive performance, and little consideration regarding the permeability of magnetostrictive material is taken into account. Actually, the permeability has noticeable influence on the ME performance, and the MEVC is inevitably connected with it.

In this paper, the equivalent circuit of a magnetostrictive/piezoelectric laminate composite (MPLC) is derived in consideration of the permeability of magnetostrictive material. The analytical model indicates that the MEVC of an MPLC is closely dependent on the permeabilty as well as the piezomagnetic coefficient. The physics lies in

*Corresponding author: Tel: +86-23-65112797

Fax:+86-23-65112797, e-mail: ymwen@cqu.edu.cn that, the magnetostrictive material is magnetized in magnetic field and produces a nonuniform demagnetizing field inside and outside it. The demagnetization is decided by the permeability of magnetostrictive material and its size $[4,5]$. The ME performances are determined by the synthesis of the applied and demagnetizing fields. Our experimental results show that the MEVC is positively connected with the permeability and the piezomagnetic coefficient of magnetostrictive material. To obtain the maximum MEVC, the permeability and the piezomagnetic coefficient of magnetostrictive material should both be taken into account in selecting the suitable magnetostrictive material.

\section{Theoretical Analysis}

2.1. Effective magnetic field in magnetostrictive layer When a magnetostrictive material is exposed to a magnetic field, considering the influence of demagnetization, the magnetic field intensity at position vector $\mathbf{r}$ can be written as

$$
\mathbf{H}_{\text {eff }}(\mathbf{r})=\mathbf{H}_{\mathrm{ap}}(\mathbf{r})+\mathbf{H}_{\mathrm{d}}(\mathbf{r})
$$

where $\mathbf{H}_{\text {eff }}(\mathbf{r})$ is the effective magnetic field with an externally applied magnetic field $\mathbf{H}_{\mathrm{ap}}(\mathbf{r}), \mathbf{H}_{\mathrm{d}}(\mathbf{r})$ is the 
magnetic field intensity due to the magnetization of the magnetostrictive material. $\mathbf{H}_{\mathrm{d}}(\mathbf{r})$ is also called the demagnetizing field, and is given by [6,7]

$$
\begin{aligned}
\mathbf{H}_{\mathrm{d}}(\mathbf{r})= & \frac{1}{4 \pi \mu_{0}} \int_{\forall} \frac{-\nabla \cdot \mathbf{J}(\mathbf{s})}{|\mathbf{r}-\mathbf{s}|^{3}}(\mathbf{r}-\mathbf{s}) d \forall(\mathbf{s}) \\
& +\frac{1}{4 \pi \mu_{0}} \int_{S} \frac{\mathbf{n} \cdot \mathbf{J}(\mathbf{s})}{|\mathbf{r}-\mathbf{s}|^{3}}(\mathbf{r}-\mathbf{s}) d S(\mathbf{s})
\end{aligned}
$$

where $\mathbf{J}(\mathbf{s})$ is the magnetic polarization distribution, $\mathbf{s}$ is the position vector of an arbitrary point inside or on the surface of magnetostrictive material. Equation (2) implies that the demagnetizing field can be considered to be caused by an induced volume magnetic charge density $\rho=-\nabla \cdot \mathbf{J}$ and an induced surface magnetic charge density $\sigma=\mathbf{n} \cdot \mathbf{J}$.

For a uniformly applied magnetic field, assuming that magnetostrictive material is uniformly magnetized, we get $\nabla \cdot \mathbf{J}=0$. This eliminates the volume integral in (2) and reduces $\mathbf{H}_{\text {eff }}(\mathbf{r})$ to $[6,8]$

$$
\mathbf{H}_{\mathrm{eff}}(\mathbf{r})=\mathbf{H}_{\mathrm{ap}}(\mathbf{r})+\frac{1}{4 \pi \mu_{0}} \int_{S} \sigma(\mathbf{s}) \frac{\mathbf{r}-\mathbf{s}}{|\mathbf{r}-\mathbf{s}|^{3}} d S(\mathbf{s})
$$

Thus, the demagnetizing field can be considered to be caused only by surface magnetic charges.

Fig. 1 illustrates that the rectangular prism located at $-c \leq x \leq c,-b \leq y \leq b$ and $-a \leq z \leq a$. If $\mathbf{H}_{a p}$ is applied along $z$-direction, a surface magnetic charge is created on its faces $z= \pm a$. In the particular case $b \rightarrow \infty$, the effective magnetic field in the $z$-direction can be written as

$$
\begin{aligned}
H_{\mathrm{eff}}= & H_{\mathrm{ap}}+\frac{\sigma}{2 \pi \mu_{0}}\left[\arctan \left(\frac{x+c}{z-a}\right)-\arctan \left(\frac{x-c}{z-a}\right)\right. \\
& \left.-\arctan \left(\frac{x+c}{z+a}\right)+\arctan \left(\frac{x-c}{z+a}\right)\right]
\end{aligned}
$$

Thus, the effective magnetic field in the $z$-direction is nonuniform.

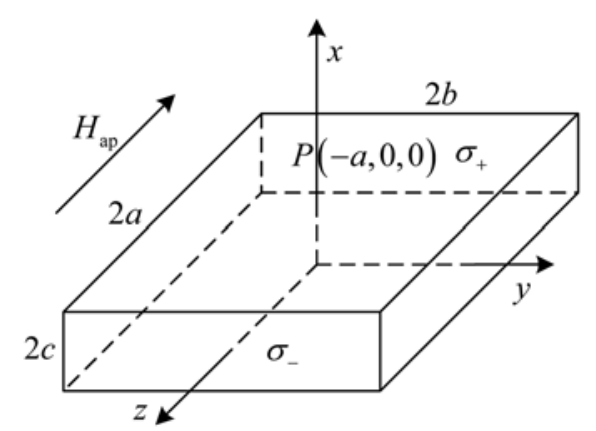

Fig. 1. The studied prism with coordinates and applied magnetic field.

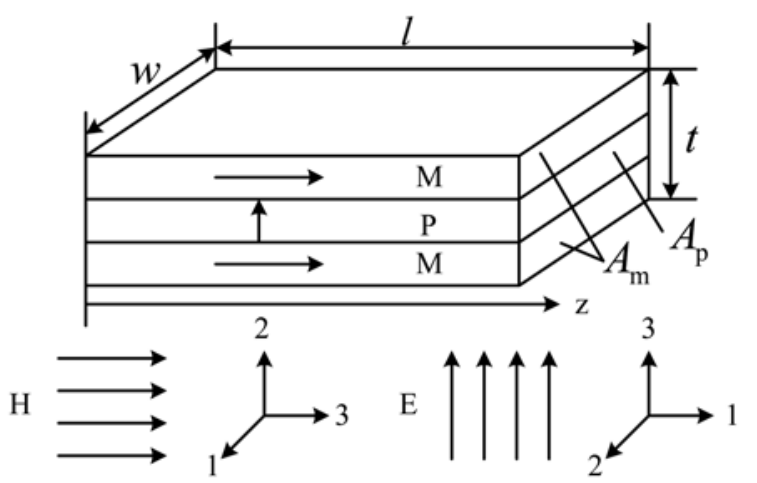

Fig. 2. Schematic of the MP laminate composite.

\subsection{Equivalent circuit}

We consider a sample in the form of a sandwich MPM laminate composite (Fig. 2). In this structure, the magnetostrictive layers are magnetized along the longitudinal direction $\mathbf{z}$ (L mode), and the piezoelectric layer is polarized in its thickness direction (T mode). When an externally applied magnetic field is applied along the longitudinal direction, a strain in magnetostrictive layers will be generated by magnetostrictive effect and transferred to the bonded piezoelectric layer. Then, the ME voltage is generated by piezoelectric effect.

Assuming that $A=2 A_{\mathrm{m}}+A_{\mathrm{p}}=t w$ is the cross-sectional area, $n=2 A_{\mathrm{m}} / A=2 t_{\mathrm{m}} / t$ is the thickness ratio, $\rho=\left(2 \rho_{\mathrm{m}} A_{\mathrm{m}}+\rho_{\mathrm{p}} A_{\mathrm{p}}\right) / A$ is the average mass density, where $t$ and $w$ denote the thickness and width of the composite; $A_{\mathrm{m}}=t_{\mathrm{m}} w$ and $A_{\mathrm{p}}=t_{\mathrm{p}} w$ are the cross-sectional area of the magnetostrictive and piezoelectric layers; and $\rho_{\mathrm{m}}$ and $\rho_{\mathrm{p}}$ are the mass densities of the magnetostrictive and piezoelectric layers, respectively.

Under LT mode, when nonuniform magnetic field is considered, the constitute equations of piezomagnetic and piezoelectric materials can be written as $[9,10]$

$$
\begin{aligned}
& S_{3 \mathrm{~m}}=S_{33}^{\mathrm{H}} T_{3 \mathrm{~m}}+d_{33, \mathrm{~m}} H_{3 \mathrm{eff}} \\
& B_{3 \mathrm{eff}}=d_{33, \mathrm{~m}} T_{3 \mathrm{~m}}+\mu_{33}^{\mathrm{T}} H_{3 e f f} \\
& S_{1 \mathrm{p}}=s_{11}^{\mathrm{E}} T_{1 \mathrm{p}}+d_{31, \mathrm{p}} E_{3} \\
& D_{3}=d_{31, \mathrm{p}} T_{1 \mathrm{p}}+\varepsilon_{33}^{\mathrm{T}} E_{3}
\end{aligned}
$$

where $H_{3 \text { eff }}$ and $B_{3 \text { eff }}$ are the effective magnetic field intensity and the effective magnetic flux density inside the magnetostrictive layers along $\mathbf{z} ; S_{3 \mathrm{~m}}$ and $T_{3 \mathrm{~m}}$ are the strain and stress in the magnetostrictive layers along $\mathbf{z}$; $s_{33}^{\mathrm{H}}, d_{33, \mathrm{~m}}$, and $\mu_{33}$ are the elastic compliance at constant $H$, the longitudinal piezomagnetic constant, and the magnetic permeability at constant stress in the magnetostrictive layers; $D_{3}$ and $E_{3}$ are the dielectric displacement and electric field in the piezoelectric layer along $\mathbf{z} ; S_{3 \mathrm{p}}$ and $T_{3 \mathrm{p}}$ 
are the strain and stress in the piezoelectric layer along $\mathbf{z}$; and $s_{33}^{\mathrm{E}}, d_{33, \mathrm{p}}$, and $\varepsilon_{33}$ are the elastic compliance at constant $E$, the piezoelectric constant, and the dielectric constant in the piezoelectric layer, respectively.

According the equivalent circuit method [2], we assume that $\dot{u}_{0}$ and $\dot{u}_{1}$ are the velocity of MPLC at $z=0$ and $z=l$, respectively, so the equation of motion can be written as

$$
\frac{\partial^{2} u}{\partial z^{2}}+k^{2} u=\frac{n d_{33, \mathrm{~m}}}{\rho v^{2} s_{33}^{\mathrm{H}}} \frac{\partial H_{3 \mathrm{eff}}}{\partial z}
$$

When $H_{3 \text { eff }}$ along the central axis, from (4), we get

$$
H_{3 \mathrm{eff}}^{\prime}=\left.\frac{\partial H_{3 \mathrm{eff}}}{\partial z}\right|_{x=0}=\left.\frac{\mu_{\mathrm{r}}-1}{\pi} H_{3 \mathrm{eff}}\right|_{z=0}\left[\frac{c}{z^{2}+c^{2}}-\frac{c}{(z-l)^{2}+c^{2}}\right]
$$

Hence, the general solution of (7) is

$$
\begin{aligned}
u(z)= & \frac{\dot{u}_{1} \sin k z-\dot{u}_{0} \sin k(l-z)}{j \omega \sin k l}+\left.\frac{n d_{33, \mathrm{~m}}}{k \rho v^{2} s_{33}^{\mathrm{H}}} \frac{\mu_{\mathrm{r}}-1}{\pi} H_{3 \mathrm{eff}}\right|_{z=0} \\
& \cdot[g(l)-g(0)] \frac{\cos k l}{\sin k l} \sin k z+[g(0)-g(z)] \cos k z \\
& -[h(l)-h(z)] \sin k z
\end{aligned}
$$

where

$$
\begin{aligned}
g(z)= & \int\left[\frac{c}{z^{2}+c^{2}}-\frac{c}{(z-l)^{2}+c^{2}}\right] \sin k z d z, \\
& h(z)=\int\left[\frac{c}{z^{2}+c^{2}}-\frac{c}{(z-l)^{2}+c^{2}}\right] \cos k z d z .
\end{aligned}
$$

For the MPLC shown in Fig. 2, the equivalent circuit considering nonuniform effective magnetic field can be given in Fig. 3. In this figure, $\varphi_{p}=\frac{w d_{31, \mathrm{p}}}{s_{11}^{\mathrm{E}}}, \varphi_{\mathrm{m}}=\frac{2 A_{\mathrm{m}} d_{33, \mathrm{~m}}}{s_{33}^{\mathrm{H}}}$, $\xi=\left.\frac{\mu_{\mathrm{r}}-1}{\pi} H_{3 \mathrm{eff}}\right|_{z=0}\left\{[g(0)-g(l)] \frac{\cos k l}{\sin k l}-[h(0)-h(l)]+\frac{g^{\prime}(0)}{k}\right\}$, $Z=-\frac{1}{2} j \rho v A \tan \frac{k l}{2}, C_{0}=\frac{l w \bar{\varepsilon}_{33}}{t_{\mathrm{p}}}, \bar{\varepsilon}_{33}=\varepsilon_{33}\left(1-k_{31, \mathrm{p}}^{2}\right), v^{2}=\left(\frac{n}{s_{33}^{\mathrm{H}}}+\frac{1-n}{s_{11}^{\mathrm{E}}}\right) / \rho$, $k=\omega / v, v$ is the sound velocity of the MPLC, $\omega$ is the angular frequency, $\mu_{\mathrm{r}}$ is the relative permeability of the magnetostrictive layer, and $k_{31, \mathrm{p}}$ is the electromechanical coupling coefficient of the piezoelectric layer.

Applying Ohm's law and impedance conversion method, we have

$$
\left|\frac{\varphi_{\mathrm{p}} V}{\left.\varphi_{\mathrm{m}} H_{3 \mathrm{eff}}\right|_{z=0}+\varphi_{\mathrm{m}} \xi}\right|=\left|\frac{\varphi_{\mathrm{p}}^{2}}{j \omega C_{0} Z+\varphi_{\mathrm{p}}^{2}}\right|
$$

From (4), the relationship between $\left.H_{3 \mathrm{eff}}\right|_{z=0}$ and the externally applied magnetic field along the $z$-direction $H_{3 \text { ap }}$ can be written as

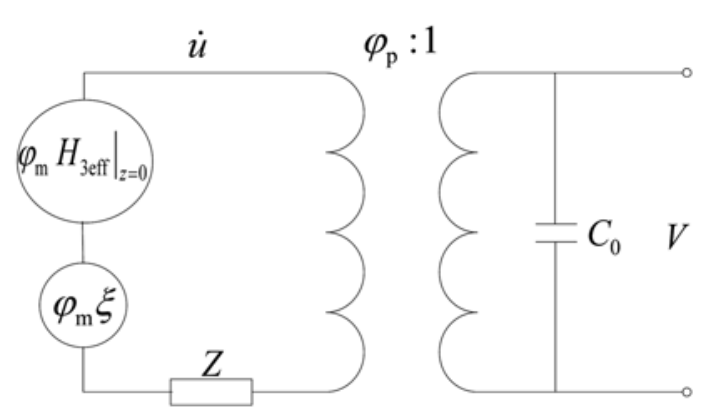

Fig. 3. Magnetoelectricity equivalent circuit.

$$
\left.H_{3 \mathrm{eff}}\right|_{z=0}=\frac{H_{3 \mathrm{ap}}}{1+\left[\arctan \left(\frac{c}{2 a}\right)+\frac{\pi}{2}\right] \frac{\mu_{\mathrm{r}}-1}{\pi}}
$$

Recognizing that the value of $\tan (k l / 2)$, in the characteristic impedance $Z$ is $\sim k l / 2$ at low frequency, and substituting (11) into (10), we obtain the MEVC as

$$
\alpha_{V}=\left|\frac{d V}{d H_{3 \mathrm{ap}}}\right|=\frac{n(1-n) t d_{31, \mathrm{p}} d_{33, \mathrm{~m}}}{\varepsilon_{33}^{\mathrm{T}}\left[n\left(1-k_{31 \mathrm{p}}^{2}\right) s_{11}^{\mathrm{E}}+(1-n) s_{33}^{\mathrm{H}}\right]} \cdot \delta
$$

where, $\delta=\frac{1+\xi \frac{\mu_{\mathrm{r}}-1}{\pi}}{1+\left[\arctan \left(\frac{t_{\mathrm{m}}}{2 l}\right)+\frac{\pi}{2}\right] \frac{\mu_{\mathrm{r}}-1}{\pi}}$ is related to the nonuniform effective magnetic field. From (12), we can see that, the MEVC of an MPLC is closely dependent on the permeability and the piezomagnetic coefficient of the magnetostrictive material.

\section{Experiment Results and Discussion}

We design and fabricate two composites of FeNi-PZT8FeNi (No.1) and Permendur-PZT8-Permendur (No.2). The dimensions of FeNi, Permendur and PZT 8 are $12 \times$ $6 \times 0.6 \mathrm{~mm}^{3}, \quad 12 \times 6 \times 0.6 \mathrm{~mm}^{3}$ and $12 \times 6 \times 0.8 \mathrm{~mm}^{3}$,

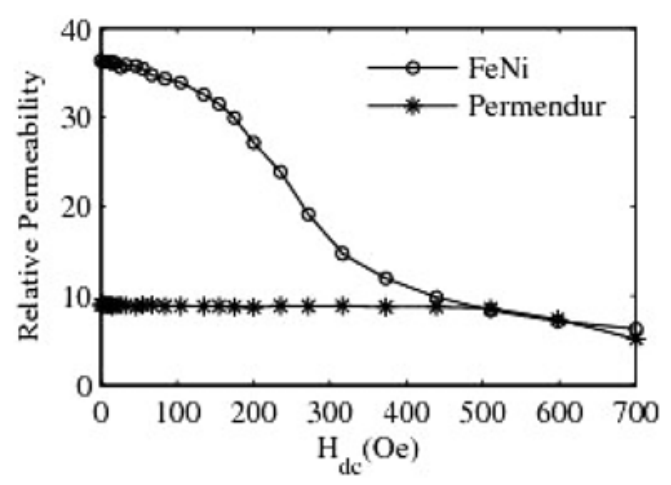

Fig. 4. The relative permeability as function of $H_{\mathrm{dc}}$ for FeNi and Permendur. 


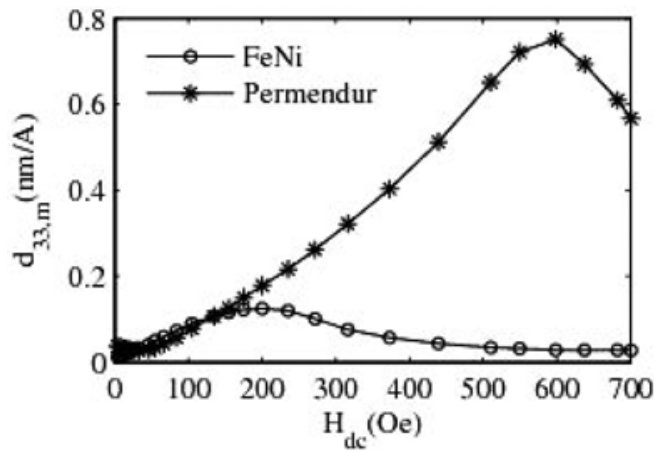

Fig. 5. The piezomagnetic coefficients $d_{33, \mathrm{~m}}$ as function of $H_{\mathrm{dc}}$ for FeNi and Permendur.

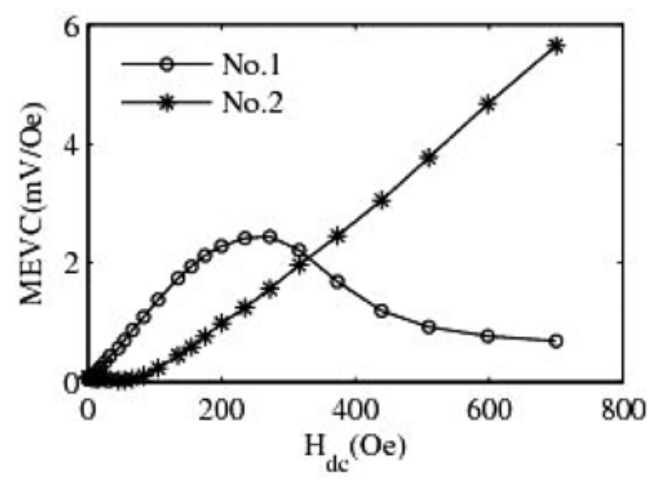

Fig. 6. MEVC as a function of $H_{\mathrm{dc}}$ at $H_{\mathrm{ac}}=0.5$ Oe for No.1 and No.2.

respectively. The relative permeabilities of $\mathrm{FeNi}$ and Permendur are measured using a method of testing the voltage of $H$ coil and $B$ coil [11]. Fig. 4 shows the relative permeabilities as function of bias magnetic field $H_{\mathrm{dc}}$ for FeNi and Permendur, respectively. Using the experimental setup (Ref. [12]), we measure the piezomagnetic coefficients $d_{33, \mathrm{~m}}$ versus $H_{\mathrm{dc}}$ for FeNi and Permendur, as shown in Fig. 5.

Fig. 6 shows MEVC as a function of $H_{\mathrm{dc}}$ at applied AC magnetic field $H_{\mathrm{ac}}=0.5$ Oe for No.1 and No.2. In the range of $0 \mathrm{Oe}<H_{\mathrm{dc}}<200 \mathrm{Oe}$, the $d_{33, \mathrm{~m}}$ of FeNi and Permendur are approximately equal, the relative permeability of FeNi is larger than that of Permendur, and the MEVC of No.1 is much higher than that of No.2. When $H_{\mathrm{dc}}>500 \mathrm{Oe}$, the $d_{33, \mathrm{~m}}$ of Permendur is larger than that of $\mathrm{FeNi}$, the relative permeability of $\mathrm{FeNi}$ and Permendur are approximately equal, and the MEVC of No.2 is much higher than that of No.1. In short, to obtain the maximum
MEVC, using different magnetostrictive materials to laminate with the same piezoelectric layer, when 0 Oe $<H_{\mathrm{dc}}<200$ Oe, FeNi is better than Permendur; when $H_{\mathrm{dc}}>500$ Oe, Permendur is better than FeNi.

\section{Conclusion}

A theoretical model including nonuniform magnetic field has been developed for MPLC. The theoretical and experimental results illustrate that the MEVC positively depends on the relative permeability and the piezomagnetic coefficient. When designing high performance $\mathrm{ME}$ devices, the magnetostrictive material with much higher permeability and much higher piezomagnetic coefficient will be more suitable.

\section{Acknowledgment}

This work is supported by the National Natural Science Foundation of China (No. 50830202, 61071042), and the Ph.D. Programs Foundation of Ministry of Education of China (No. 20100191110009).

\section{References}

[1] G. Srinivasan, Annu. Rev. Mater. Res. 40, 153 (2010).

[2] S. X. Dong, J.-F. Li, and D. Viehland, J. Mater. Sci. 41, 97 (2006).

[3] F. Yang, Y. M. Wen, P. Li, M. Zheng, and L. X. Bian, Sens. Actuators A 141, 129 (2008).

[4] A. Aharoni, J. Appl. Phys. 83, 3432 (1998).

[5] D. X. Chen, E. Pardo, and A. Sanchez, IEEE Trans. Magn. 41, 2077 (2005).

[6] S. M. Dutta, F. Ghorbel, and R. Stanley, IEEE Trans. Magn. 45, 1959 (2009).

[7] J. Z. Yi, Magnetic Field Calculation and Magnetic Circuit Design, Chengdu Electronic Information Engineering College Press, Chengdu (1987) pp. 16 20.

[8] X. Z. Dai et al., Acta Phys. Sin. 59, 2137 (2010).

[9] C. M. Chang and G. P. Carman, Phys. Rev. B 76, 134116 (2007).

[10] J. Yang, Y. Wen, P. Li, and X. Dai, Proc. Power MEMS 2009, Washington (2009) pp. 352 355.

[11] B. W. Wang et al., Magnetostictive Materials and Devices, Metallurgical Industry Press, Beijing (2008) p. 158.

[12] L. X. Bian, Y. M. Wen, P. Li, Q. L. Gao, and X. X. Liu, J. Magnetics 14, 66 (2009). 